\title{
The Development of a Gold-Based Activator for Autocatalytic Plating
}

\author{
Michael C. Stuart \\ Fulmer Research Institute Ltd., Stoke Poges, England
}

\begin{abstract}
The industrial activators or catalysts used to date to promote the electroless plating of plastics have been mostly based on palladium and proprietary in nature. Gold-based catalysts are also effective, however, and in the present article the author describes a colloidal gold activator which is easy to prepare and use. A seeding procedure is employed in its preparation and is significant in determining both its stability and performance.
\end{abstract}

The electroless or autocatalytic plating process makes possible the pre-coating of non-conducting surfaces with an adherent metallic film of sufficient coverage and thickness (about $1 \mu \mathrm{m}$ ) to serve as a cathode for subsequent conventional electroplating.

In principle, the process can be used for coating any surface, conducting or insulating, but its main application is in the plating of plastics. In particular, commercial electroless processes have been developed for use with acrylonitrile-butadiene-styrene (ABS) polymers. They have also been used with polypropylenes but they are not as successful with plastics of this type as they are with ABS polymers. Most practical applications of electroless plating involve the deposition of one of the more reducible metals, such as copper or nickel, on these plastics.

The electroless plating process as it is applied to non-conductors involves most, and sometimes all, of the following stages: pre-cleaning, etching, neutralizing, sensitizing, activating, accelerating and autocatalytic plating. Controlled water rinses are interposed between the stages. There is a considerable body of information on the variables affecting each of these steps (1).

The activating stage in autocatalytic plating of nonconductors consists of immersion of the object to be plated in a noble metal-tin hydrosol, followed by a rinse in de-ionized water. It is followed in the accelerating stage by immersion of the substrate in a solution which dissolves the protective colloid and leaves the surface densely seeded with noble metal nuclei. The two steps are interrelated. The reagent in the first step is usually referred to as the activator and that in the second as the accelerator.

The function of the noble metal nuclei is to initiate the autocatalytic deposition of metal when the substrate is next immersed in the electroless plating solution, which contains essentially the metal to be plated and a reducing agent. The precise mechanism of this initiation or activation reaction is not well understood although a number of theories have been suggested ( 2 to 5 ). The end result, however, is the reduction of the copper or nickel ions in the plating bath to copper or nickel in such a manner that the deposited metal atoms agglomerate to form a continuous metal layer on the activated substrate.

In current industrial practice, activators containing colloidal palladium are used for electroless plating. These preparations have been developed to a high degree of technical excellence and reliability over many years. Most are, however, proprietary products and the development of alternatives to these activators could be of great advantage. Gold is not generally regarded as an important catalyst although it has been shown that the autocatalytic plating reactions of nickel and copper can be initiated by suitably formulated preparations which contain gold as the active ingredient $(6,7,8)$. On this basis, a systematic investigation was undertaken with the purpose of devising a working gold-based activator for autocatalytic plating.

\section{Experimental Procedures}

A plating line was used for this work which was capable of treating plating grades of both ABS and polypropylene plastics. The treatment steps employed proprietary products used according to the manufacturers' instructions. Experimental preparations were used at the activating step only.

The activator investigated was a gold-stannic oxide hydrosol suspended in a strongly acidic aqueous solution of stannous chloride, and the aim of the work was to optimise the concentration of the reagents used in its preparation so as to obtain an effective and stable activator as judged by the quality of the subsequent electroplate. 
A preliminary survey was made of the effect of concentration variables, using a factorial experiment. The following concentrations of reagents were investigated:

moles $/ 1$

Gold $\quad 0.00025,0.0005,0.001,0.002$

Stannous chloride $0.04,0.08,0.16,0.32$

Hydrochloric acid $0.5,1.5,2.5,3.5,5.0$

The activators were prepared by adding the gold in the form of a chloroauric acid solution to a previously prepared solution containing the requisite amounts of hydrochloric acid and stannous chloride. The gold solution was added quickly, while stirring with a glass rod, which resulted in the immediate formation of a dark brown hydrosol. After preparation the activators were allowed to stand for several days in sealed containers before testing.

Those activators containing 0.00025 and 0.0005 mole/l of gold gave poor results in plating tests partly because the colloidal particles agglomerated and settled out rapidly. However, at higher gold concentrations and at a stannous chloride concentration of 0.32 mole/1, stable activators were obtained with acid.concentrations greater than 1.5 mole/l. With these activators, subsequent plating of nickel or copper resulted in even and complete covering of the test samples. These solutions also performed satisfactorily when diluted ten-fold.

As a result of this preliminary work the gold concentration was fixed at $0.0025 \mathrm{~mole} / \mathrm{l}$, a value which was selected to give maximum stability to the hydrosol. The optimum concentrations of hydrochloric acid and stannous chloride were then investigated in a further series of experiments.

\section{Hydrochloric Acid Concentration}

Activators were prepared by the method described above, using the following concentrations of reagents:

$\begin{array}{ll} & \text { moles/l } \\ \text { Gold } & 0.0025 \\ \text { Stannous chloride } & 1.58 \\ \text { Hydrochloric acid } & 0.49,1.47,1.96,2.45,2.94,3.92, \\ & 5.88\end{array}$

These activators were diluted ten-fold with hydrochloric acid of the same concentration as that used in the original make-up and were then tested at $20^{\circ} \mathrm{C}$. Within 24 hours, a precipitate formed in those with the lowest acid concentration. Those with higher acid concentrations were stable for longer than 40 days. Plating tests revealed no significant variation in activity of diluted working solutions prepared from concentrates with acid concentration from 1.47 to 5.88 moles $/ 1$. Thus, it was decided to adopt a value of $2.45 \mathrm{moles} / 1$ as the standard acid concentration.

\section{Stannous Chloride Concentration}

Stannous chloride, as it is usually supplied, contains a small but significant amount of stannic oxide present as an impurity. This 'impurity' is essential to the formation of a stable hydrosol. The commercial grade of stannous chloride used in this work contained from 6 to 8 per cent of its tin in stannic form.

Activator concentrates were prepared according to the following formulations:

$\begin{array}{ll} & \text { moles } / 1 \\ \text { Gold } & 0.0025 \\ \text { Stannous chloride } & 2.45 \\ \text { Hydrochloric acid } & 0.79,1.48,3.16\end{array}$

To give working strength activators, the above concentrates were diluted by factors of $10,30,50$ and 100 with a solution of 2.45 moles/l of hydrochloric acid. The results of using these for nickel plating tests as well as their stability are given in Table I. It can be seen that the activator is effective at all stannous concentrations provided it is not diluted further than tenfold. Dilution beyond this results in a tendency for effectiveness to decrease at the higher tin/gold ratios. The stability of the solution also decreases with dilution but this effect is less marked for the higher tin/gold ratios.

As a result of these experiments, it was decided to set the concentration of the ingredients of the activator concentrate at 0.0025 gold/1.58 stannous chloride/2.94 hydrochloric acid moles/1.

Table I

Influence of the Tin/Gold Molar Ratio and the Stannous Chloride Concentration on the Effectiveness and Life of Activators for Electroless Nickel Plating

\begin{tabular}{|c|c|c|c|}
\hline $\begin{array}{l}\text { Tin/gold } \\
\text { molar } \\
\text { ratio }\end{array}$ & $\begin{array}{l}\text { Stannous } \\
\text { chloride } \\
\text { concentra- } \\
\text { tion } \\
\text { moles } / 1\end{array}$ & $\begin{array}{c}\text { Nickel- } \\
\text { plated } \\
\text { area* } \\
\\
\%\end{array}$ & $\begin{array}{c}\text { Time for } \\
\text { precipita- } \\
\text { tion } \\
\text { days }\end{array}$ \\
\hline 1264 & 3.160 & 100 & 70 \\
\hline 632 & 1.580 & 100 & 70 \\
\hline 316 & 0.790 & 100 & 70 \\
\hline 1264 & 0.320 & 100 & 70 \\
\hline 632 & 0.158 & 100 & 35 \\
\hline 1264 & 0.105 & 80 & 33 \\
\hline 316 & 0.079 & 100 & 19 \\
\hline 1264 & 0.063 & 25 & 17 \\
\hline 632 & 0.053 & 75 & 20 \\
\hline 1264 & 0.032 & 0 & 3 \\
\hline 632 & 0.032 & 75 & 12 \\
\hline 316 & 0.026 & 100 & 12 \\
\hline 632 & 0.016 & 25 & 3 \\
\hline 316 & 0.016 & 100 & 6 \\
\hline 316 & 0.008 & 25 & 3 \\
\hline \multicolumn{4}{|c|}{ *Percentage area plated after 2 minutes activation } \\
\hline
\end{tabular}


Table II

Influence of the Seeding Procedure and the Activation Time on the Effectiveness of Colloidal Gold Activators for Electroless Nickel Plating

\begin{tabular}{c|c|r|r|r|r}
\hline & & \multicolumn{3}{|c}{ Nickel plated area } \\
Concentrate & \multirow{2}{*}{ Dilution } & \multicolumn{4}{|c}{} \\
\cline { 3 - 6 } & & \multicolumn{4}{|c}{ Activation time } \\
& & 60 & 45 & 30 & 15 \\
\hline A & 10 & 95 & - & - & - \\
B & $\prime \prime$ & 100 & 100 & 90 & 70 \\
C & $\prime \prime$ & 100 & 100 & 95 & 90 \\
D & $\prime \prime$ & 100 & 100 & 100 & 100 \\
E & $\prime \prime$ & 100 & 100 & 100 & 100 \\
F & $\prime \prime$ & 100 & 100 & 100 & 95 \\
G & $\prime \prime$ & 100 & 100 & 100 & 100 \\
A & 20 & 90 & - & - & - \\
B & $\prime \prime$ & 90 & 70 & 50 & 10 \\
C & $\prime \prime$ & 95 & - & 90 & - \\
D & $\prime \prime$ & 100 & 100 & 95 & 100 \\
E & $\prime \prime$ & 100 & 100 & 100 & 95 \\
F & $\prime \prime$ & 100 & 100 & 95 & 95 \\
G & $\prime \prime$ & 100 & 100 & 95 & 90
\end{tabular}

\section{The Effect of Seeding}

During the preliminary work it became clear that the method of preparation of the activator, in particular the rate and temperature of mixing, were important parameters having an influence on the stability and the effectiveness of the activator in promoting plating. The preferred method was to stir the ingredients slowly at a temperature between 90 and $100^{\circ} \mathrm{C}$. When examined by transmission electron microscopy, the gold particles in the activator were seen to be larger in the more active preparations, having a mean diameter about $100 \AA$.

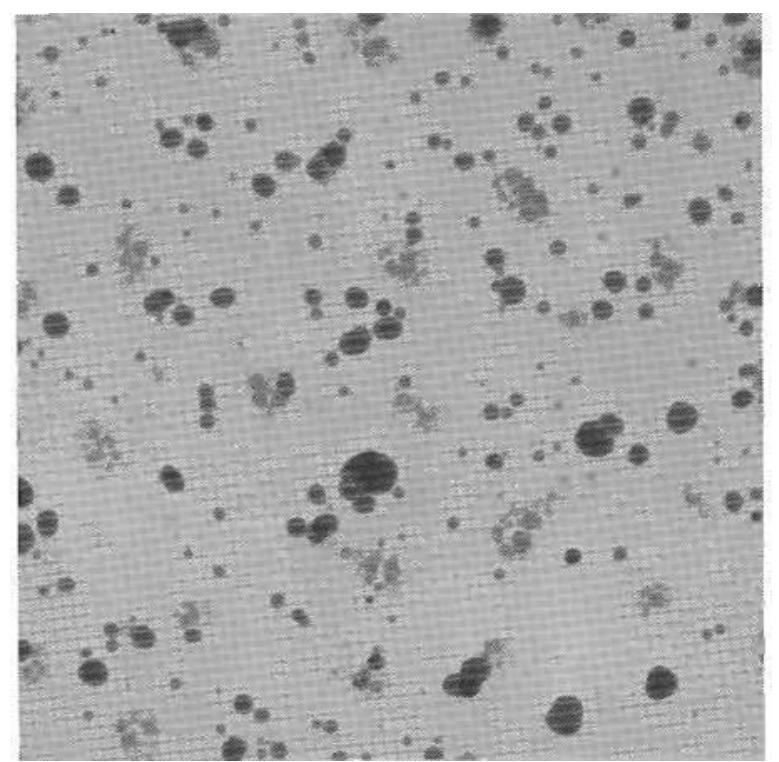

This observation of an apparent relationship between increasing particle size and catalytic effectiveness led to a series of experiments aimed at imcreasing the size of the gold particles by controlled nucleation, since one factor governing the size of particles must be the number and size of the nuclei present in the initial stage of colloid formation. It was predicted that a previously prepared activator could be used as a source of nuclei which would grow preferentially when introduced during the preparation of a new concentrate.

The procedure used to test this hypothesis was to make an initial concentrate of 1.58 stannous chloride/2.94 hydrochloric acid moles/1. One litre of this original stannous solution (OS) was heated to $90^{\circ} \mathrm{C}$ and to it was added a solution of 0.0025 mole of chloroauric acid $\left(\mathrm{HAuCl}_{4} \cdot 3 \mathrm{H}_{2} \mathrm{O}\right)$ in $5 \mathrm{ml}$ of water. A dark brown concentrated activator $(\mathrm{A})$ was produced.

To an identical quantity of stannous solution (OS) at $90^{\circ} \mathrm{C}$ was added $90 \mathrm{ml}$ of activator concentrate (A) followed by sufficient of a solution of chloroauric acid in $5 \mathrm{ml}$ of water to raise the gold concentration to $0.0025 \mathrm{~mole} / 1 \mathrm{l}$ thus forming activator concentrate (B).

The above step was repeated using concentrate (B) to seed the basic stannous solution (OS), then again raising the gold concentrate to 0.0025 mole/l to produce a further concentrate $(C)$ and so on until step $(G)$ was reached. Activator concentrates (A) to $(\mathrm{G})$ were diluted for use by the addition of a $2.94 \mathrm{moles} / 1 \mathrm{solu}$ tion of hydrochloric acid in water. Working solutions of two strengths were prepared, a ten-fold dilution to $0.00025 \mathrm{~mole} / \mathrm{l}$ of gold and a twenty-fold dilution of $0.000125 \mathrm{~mole} / 1$ of gold. Both were tested by producing autocatalytic nickel deposits on ABS plastic sheet. The results are summarised in Table II.

The improvement in terms of coverage brought about by the first seeding step, which resulted in concentrate (B), was sufficient to mask any improvement resulting from subsequent seeding steps. However, by reducing the activating time it was possible to produce evidence of an increase in activation when using a working activator from concentrate (E) compared with (B). A flawless product was obtained.

Electron micrograph showing the fine colloidal nature of the gold-based autocatalytic plating activator 


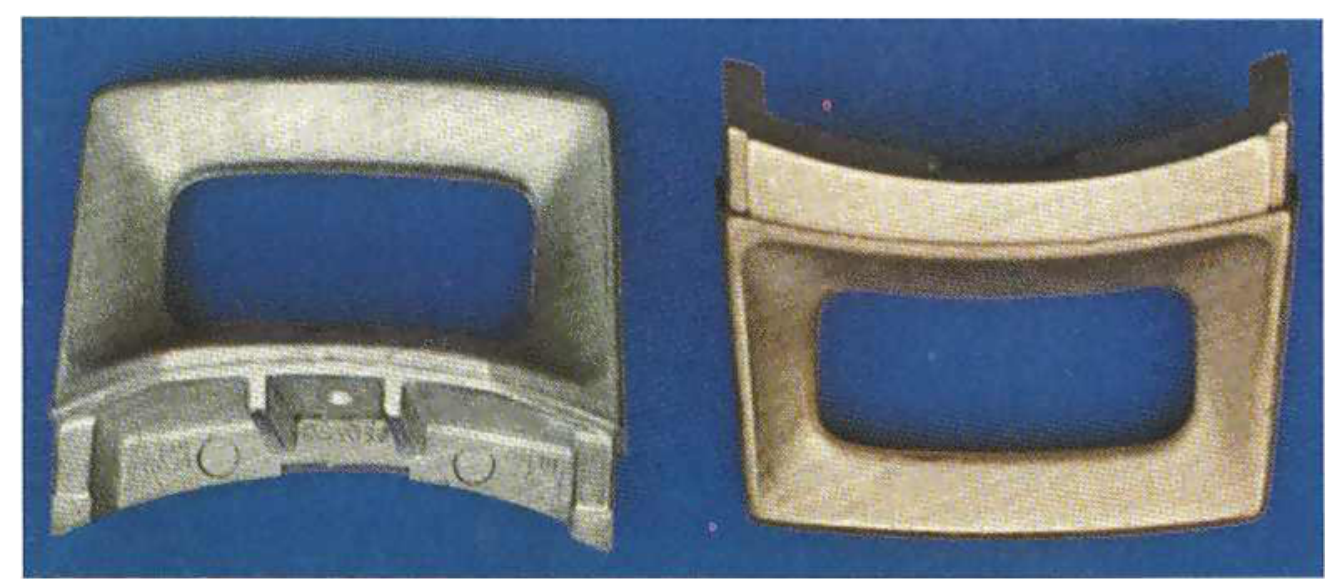

ABS polymer panels coated with nickel and copper using the gold-based autocatalytic plating activator developed at Fulmer Research Institute Ltd.

Further experiments were made to determine the minimum quantity of seeding which was necessary. Amounts of seeding varying from 1 to 20 per cent were used during the preparation of new concentrates. In subsequent plating with electroless nickel, all the seeded activators gave 100 per cent coverage of ABS test panels whereas the unseeded activator gave only 50 per cent coverage under identical conditions. It is considered that as little as 1 per cent of the seeding solutions is adequate to give improvement compared with unseeded activator.

\section{Conclusions}

An activator concentrate containing colloidal gold was successfully developed for the autocatalytic plating of plastics by copper and nickel. The recommended composition for the aqueous concentrate is $0.0025 \mathrm{~mole} / 1$ gold, $0.158 \mathrm{~mole} / 1$ stannous chloride and 2.94 moles/ 1 hydrochloric acid. The activator can be easily prepared and used, even by unskilled operators. It is very stable, both in the concentrated form and when diluted ten-fold with 2.94 moles $/ 1$ hydrochloric acid to give a working strength activator. The performance and stability of the activator are greatly improved by following the seeding procedure described.

\section{Acknowledgements}

Thanks are expressed to the Chamber of Mines of South Africa for sponsoring the work described in this paper. Thanks too are given to Dr. W. S. Rapson and Dr. T. Groenewald for their interest and advice.

\section{References}

1 W. Goldie, 'Metallic Coating of Plastics', Electrochemical Publications Ltd., Hatch End, Middlesex, 1968

2 R. M. Lukes, Plating, 1964, 51, 1066-1068

3 A. Brenner and G. E. Riddell, Proc. Am. Electroplat. Soc., $1946,16,23-27$

4 G. Salvage and P. Cavollotti, Plating, 1972, 59, (7), 665-667

5 V. Gouda, S. Shawki and H. El-Tawil, Met. Finish., 1972, 5, $77-81$

6 E. B. Saubestre, U.S. Patent 2,872,359, 1959

7 H. Narcus, Proc. Am. Electroplat. Soc., 1956, 43, 157-161

8 E. B. Saubestre, Met. Finish., 1962, 60, (6), 67-73; (7), 49-53; (8), 45-49, 52; (9), 59-63 